\title{
Theory of cosmic ray modulation
}

\author{
Stefan E. S. Ferreira \\ Unit for Space Physics, North-West University, 2520 Potchefstroom, South Africa \\ email: Stefan.Ferreira@nwu.ac.za
}

\begin{abstract}
This work aims to give a brief overview on the topic of cosmic ray modulation in the heliosphere. The heliosphere, heliospheric magnetic field, transport parameters and the transport equation together with modulation models, which solve this equation in various degree of complexity, are briefly discussed. Results from these models are then presented where first it is shown how cosmic rays are globally distributed in an asymmetrical heliosphere which results from the relative motion between the local interstellar medium and the Sun. Next the focus shifts to low-energy Jovian electrons. The intensities of these electrons, which originate from a point source in the inner heliosphere, exhibit a unique three-dimensional spiral structure where most of the particles are transported along the magnetic field lines. Time-dependent modulation is also discussed where it is shown how drift effects together with propagating diffusion barriers are responsible for modulation over a solar cycle.
\end{abstract}

Keywords. (ISM:) cosmic rays, (Sun:) solar wind, plasmas, turbulence.

\section{Introduction}

Cosmic rays, when either entering the heliosphere due to their galactic origin, or being produced inside, are subjected to different modulation processes. These include diffusion, convection, energy changes and drifts (see Potgieter 1998; Fichtner 2005, for an overview). The Sun is the source of the heliosphere and as the activity of the Sun periodically changes from maximum to solar minimum conditions (every 11 years) we also observe solar cycle related changes in cosmic ray intensities due to changes in the modulation environment. As these particles travel to Earth they sample the different heliospheric conditions and can therefore provide valuable information about the outer regions of our heliosphere and even distances beyond (Lee \& Fichtner 2001).

Apart from a wide range of neutron monitors on Earth (e.g., Moraal et al. 2000) which have been measuring cosmic ray intensities over the past few decades, there is also a fleet of spacecraft (like the Helios, Pioneers, Voyagers, Ulysses and PAMELA missions, to name a few) who measure cosmic rays at different energies and positions. Measurements from these missions have increased our knowledge, not only on cosmic ray transport, but also on the details of the background plasma environment in our local astrosphere. These observations have stimulated, to name a few, the development of different state of the art modulation (e.g., Fisk 1971; Kota \& Jokipii 1983; le Roux \& Potgieter 1995; Hattingh \& Burger 1995) and heliospheric (e.g., Baranov \& Malama 1995; Pauls \& Zank 1996; Washimi \& Tanaka 1996; Fahr et al. 2000; Pogorelov et al. 2006) models, theories on particle transport (e.g., Jokipii 1966; Bieber et al. 1994; Burger et al. 2000; Lerche \& Schlickeiser 2001; Teufel \& Schlickeiser 2002), turbulence in the solar wind (e.g., Bieber et al. 2004; Matthaeus et al. 2003), models of the heliospheric magnetic field (e.g., Parker 1958; Fisk 1996; Burger 2005), detailed theories on acceleration at shocks (e.g., Bell 1978; Blandford \& Ostriker 1978) as well as continuous acceleration in the inner heliosheath (e.g., Kallenbach et al. 2005; Fisk \& Gloeckler 2006) etc. 
Furthermore, high-energy galactic cosmic rays may also influence the climate on Earth. Although controversial, Friis-Christensen \& Svensmark (1997) and Svensmark (1998) found a correlation between cosmic ray intensity and global cloud coverage on the 11-year time scale of solar activity. There also seems to be a correlation on much longer timescales due to influences by the interstellar environment or spiral arm crossings (Shaviv 2003; Shaviv \& Veizer 2003). See e.g. Scherer et al. (2006) for more details. Also of importance is the significant danger these high energy particles may have on future human space exploration (Parker 2005).

While the galactic cosmic rays originate outside the solar system due to acceleration at astrophysical shocks, the anomalous cosmic rays (e.g., Fisk et al. 1974) are accelerated inside the heliosphere. Voyager measurements (Decker et al. 2005; Stone et al. 2005) showed that the flux of anomalous cosmic rays in the heliosheath is unexpectedly high compared to expectations before Voyager 1 reached the shock. This might be due to particle acceleration at the flanks of the heliosphere (McComas \& Schwadron 2006), dynamic effects (Florinski \& Zank 2006) or due to additional continuous acceleration beyond the termination shock (Langner et al. 2006; Kallenbach et al. 2005; Fisk \& Gloeckler 2006; Ferreira et al. 2007). Combining this finding with different model results for astrosphere immersed in different interstellar environments Scherer et al. (2008) showed that the astrospheric anomalous cosmic ray fluxes of solar-type stars can be a hundred times higher than thought earlier and, consequently, their total contribution to the lower end of the interstellar spectrum can be significant.

The aspects discussed above illustrate the importance of understanding the details of cosmic ray modulation in our heliosphere. With this a detailed understanding of the modulation environment is also necessary. This work will now briefly discuss some different but closely related topics related to cosmic ray transport.

\section{The transport equation and modulation models}

Cosmic ray modulation in the heliosphere can be described by a transport equation (Parker 1965) (see also work done by Gleeson \& Axford 1967; Forman et al. 1974; Forman \& Jokipii 1978; Webb \& Gleeson 1979; Moraal \& Potgieter 1982):

$$
\begin{aligned}
& \frac{\partial f}{\partial t}=-\left(\vec{V}+\left\langle\overrightarrow{v_{D}}\right\rangle\right) \cdot \nabla f+\nabla \cdot\left(\vec{K}_{S} \cdot \nabla f\right) \\
& \quad+\frac{1}{3}(\nabla \cdot \vec{V}) \frac{\partial f}{\partial \ln P}+\frac{1}{P^{2}} \frac{\partial}{\partial P}\left(P^{2} D \frac{\partial f}{\partial P}\right)+Q .
\end{aligned}
$$

Here $t$ is the time, $P$ is rigidity, $Q$ is any particle sources inside the heliosphere, $\vec{V}$ is the solar wind velocity, $\vec{K}_{S}$ is the diffusion tensor (discussed below) and $\left\langle\overrightarrow{v_{D}}\right\rangle$ the averaged guiding center drift velocity (e.g., Burger et al. 2000; Stawicki 2005b) for a near isotropic distribution function $f$ and $D$ a momentum diffusion coefficient. This equation can be solved numerically in various degrees of complexity in so-called modulation models (e.g., Fisk 1971; Kota \& Jokipii 1983; le Roux \& Potgieter 1990; Hattingh \& Burger 1995; Steenberg \& Moraal 1996; le Roux \& Fichtner 1999; Florinski et al. 2003; Langner \& Potgieter 2005; Ferreira \& Scherer 2006; Zhang 2006) and below some results from these models are presented.

Of primary importance to cosmic ray modulation is the coupling of the transport parameters to the background field and magnetic turbulence (see e.g., Bieber et al. 2004; Matthaeus et al. 2003; Shalchi et al. 2008). Of particular interest is determing the coefficients in $\vec{K}_{S}$. These can be theoretically calculated from plasma and magnetic field observations using either turbulence theory (e.g., Bieber et al. 1994) or the theory of 
particle wave interaction (e.g., Lerche \& Schlickeiser 2001). In both of these approaches the parallel mean free path can be computed using quasi linear theory (e.g., Jokipii 1966; Bieber et al. 1994; Burger et al. 2000; Teufel \& Schlickeiser 2002) or extensions of the latter (e.g., le Roux et al. 2005).

For the perpendicular diffusions it was shown via simulations that these may scale as the parallel coefficient (le Roux et al. 1999; Giacalone \& Jokipii 1999; Qin et al. 2002). Recent theoretical advances in perpendicular diffusion include: A nonlinear theory of the perpendicular diffusion of charged particles which include the influence of parallel scattering and dynamical turbulence (Matthaeus et al. 2003; Shalchi et al. 2004; Shalchi 2006). A nonlinear description for perpendicular particle diffusion in strong electromagnetic fluctuations using the fundamental Newton Lorentz equation (Stawicki 2005a). An equation describing compound and perpendicular diffusion of cosmic rays and random walk of the field lines (Webb et al. 2006). See also Minnie et al. (2005, 2007) for recent results and incorporation of theories into modulation models.

\section{The geometry of the heliosphere and flow profiles of the solar wind plasma inside}

The solar wind is the source of the heliosphere. However, interstellar space is not empty and contains matter in the form of the local interstellar medium (LISM). As the heliosphere moves through the LISM it forces the plasma component to flow around it. At some distance the interaction of the LISM and the solar wind causes the supersonic solar wind to decrease to subsonic speeds and a shock is created, called the solar wind termination shock. At larger distances a contact surface is eventually reached, called the heliopause. This separates the solar wind from the interstellar material. Depending on the interstellar conditions there may also be a bow shock around the heliosphere. Concerning the heliospheric geometry and global magnetic field, valuable modeling efforts have been done by Holzer (1989); Suess (1990); Pauls \& Zank (1996, 1997); Fahr et al. (2000); Scherer \& Fahr (2003b,a); Zank \& Müller (2003); Izmodenov et al. (2005); Borrmann \& Fichtner (2005); Pogorelov et al. (2006); Opher et al. (2006).

The heliosphere, resulting under the relative motion between the LISM and the Sun has an asymmetric structure, for instance the position of the termination shock in the tail region is related to the position in the nose through the relation found by Müller et al. $(2006)$ as $r_{T S, \text { tail }}=(2.08 \pm 0.04) r_{T S, \text { nose }}$ with the position of the heliopause in the nose related to the position of the termination shock as $r_{H P}=(1.39 \pm 0.01) r_{T S, n o s e}$. In addition, the heliosphere is also more elongated in the poleward directions because of the latitudinal variation of the solar wind momentum flux (McComas et al. 2001). This quantity increases by a factor of $\sim 1.5$ from the equatorial regions toward the poles, suggesting a poleward elongated termination shock. Nevertheless, the mass flux is reported to be fairly constant with latitude and over a solar cycle (McComas et al. 2003), while the dynamic pressure is changing, leading to a more pronounced asymmetry during solar minimum conditions. The effect of a changing solar wind speed over the poles on the heliospheric geometry are illustrated in Fig. 1, which shows the heliosphere in the nose regions in terms of solar wind speed and density for a isotropic wind (solar maximum) and an anisotropic wind (solar minimum). The most important feature shown here, from a cosmic ray modulation point of view, is that as solar activity changes the termination shock moves, especially at the polar and tail regions. See e.g., Scherer \& Ferreira (2005) for dynamic modeling results.

In Figure 2 (From Langner \& Potgieter 2005) the distribution of cosmic rays in an asymmetrical heliosphere is shown for minimum and moderate solar activity. Compared 
to a theoretical symmetrical case, the asymmetry of the heliosphere results in insignificant effects for the $A>0$ (protons drift in from the polar regions), but for the $A<0$ cycle (protons drift in along the current sheet) the intensities may differ by at least a factor of 1.5 for most of the heliosphere, although in the tail region a factor of 3.5 is evident. As shown in Figure 2 protons are redistributed when the heliosphere is asymmetrically bounded compared to a symmetrical heliosphere (see Figure 7 of Langner \& Potgieter 2005). Concerning the inner heliosphere not much evidence can be found in the nose-tail asymmetry when cosmic rays are considered. Also note that the intensities of accelerated particles at the shock differs between the nose and tail region. This is related to the fact that the position of the heliopause in the tail region is increased, causing a smaller population of particles to be accelerated. Also not known is the difference in the continuous acceleration processes between the nose.

\section{The heliospheric magnetic field}

Embedded in the solar wind is the Sun's magnetic field wounded up in a spiral and transported with the solar wind into space forming the heliospheric magnetic field (Parker 1958). This field determines the passage of charged particles, like cosmic rays in our heliosphere, changing their intensities with time and as a function of energy and position. A very unique signature of this spiral can be observed in low-energy electron intensities. At these energies the Jovian magnetosphere is the dominant source of electrons (Simpson et al. 1974; Chenette et al. 1974) in the inner heliosphere up to 10 AU (e.g., Ferreira et al. 2001). Shown in Figure 3 is model calculations by Ferreira et al. (2001) showing the three-dimensional distribution of Jovian electrons at different polar angles, as indicated. In the left panel, signatures of the Parker spiral is clearly visible in the intensities because of the dominance of parallel diffusion in the inner heliospheric regions.

Concerning the inner heliosphere, deviations from a pure Parker-type field are expected at certain latitudes (Fisk 1996). In a Fisk-type field, magnetic field lines exhibit extensive excursions in heliographic latitude, and this has been cited as a possible explanation for
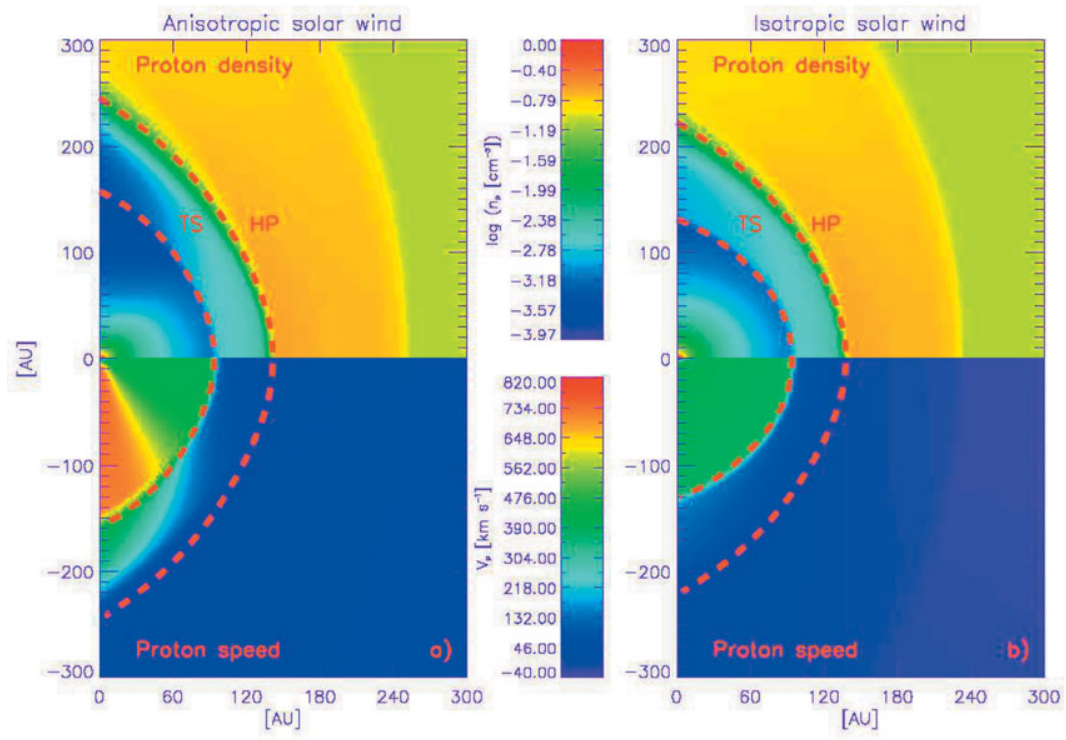

Figure 1. The heliosphere in the nose regions in terms of solar wind speed (top) and density (bottom) for an isotropic wind (solar maximum) and an anisotropic wind (solar minimum) 

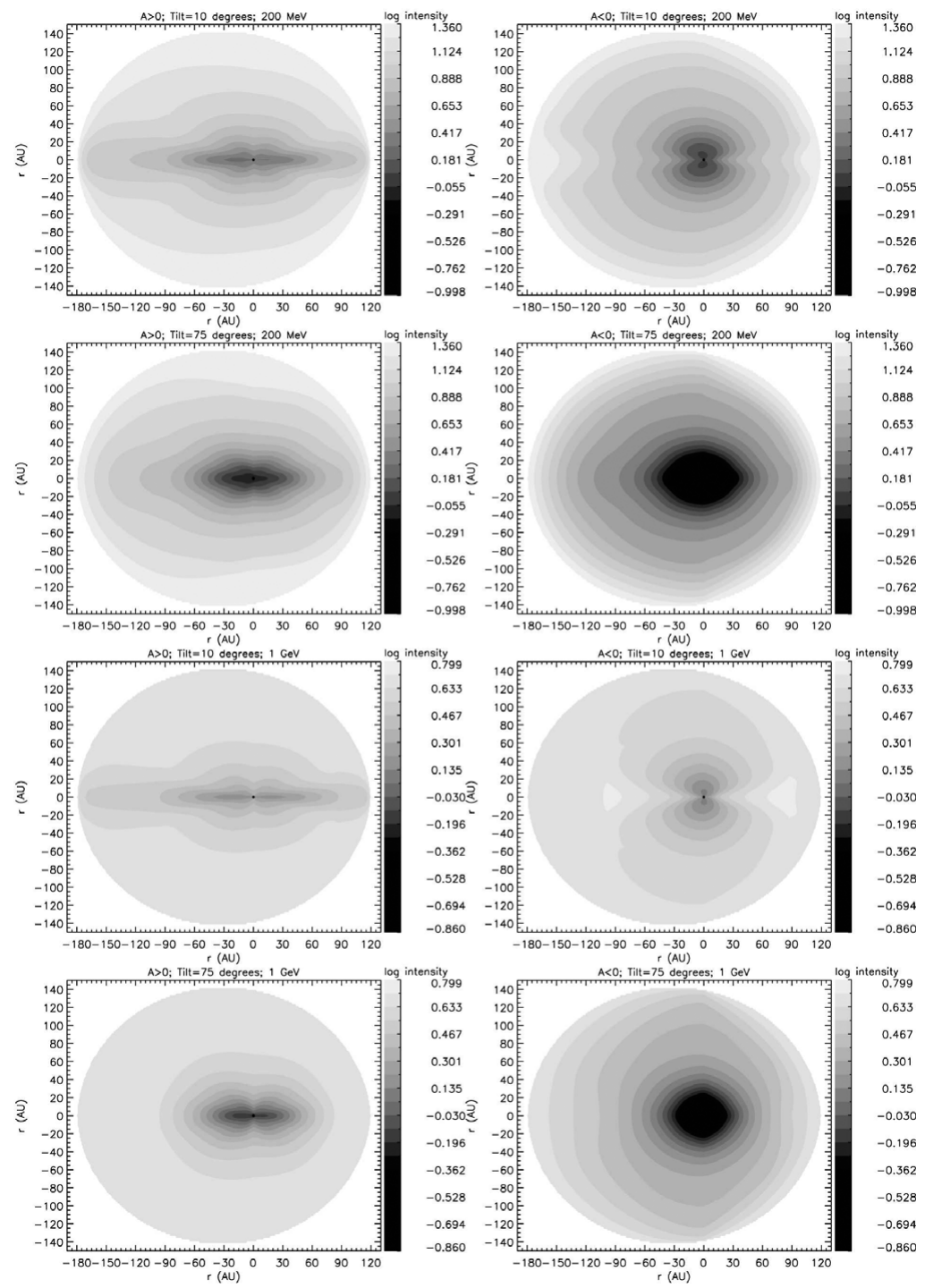

Figure 2. Proton intensity contours in the meridional plane at $200 \mathrm{MeV}$ (top four panels) and $1 \mathrm{GeV}$ (bottom four panels) for an asymmetrical heliosphere for solar minimum (tilt 10 degrees) and moderate solar maximum conditions (tilt 75 degrees), and for the $A>0$ (left) and $A<0$ (right) polarity cycles. Note that the legend of the contours corresponds to the exponent of the base 10 on a logarithmic scale and that the scaling differs for the $200 \mathrm{MeV}$ and $1 \mathrm{GeV}$ plots. (from Langner \& Potgieter 2005).

recurrent energetic particle events observed by the Ulysses spacecraft at high latitudes (see, e.g., Simpson et al. 1995; Zhang 1997; Paizis et al. 1999), as well as the smaller than expected cosmic-ray intensities observed at high latitudes (Simpson et al. 1996).

The Fisk field and the physics behind it have been discussed in a series of papers (see, e.g., Fisk \& Schwadron 2001, and references therein) where it is assumed that the polar coronal hole is symmetric with respect to the solar magnetic axis, and that the magnetic field expands nonradially. The footpoints of the magnetic field lines anchored in the photosphere experience differential rotation. Then, if the magnetic axis of the 

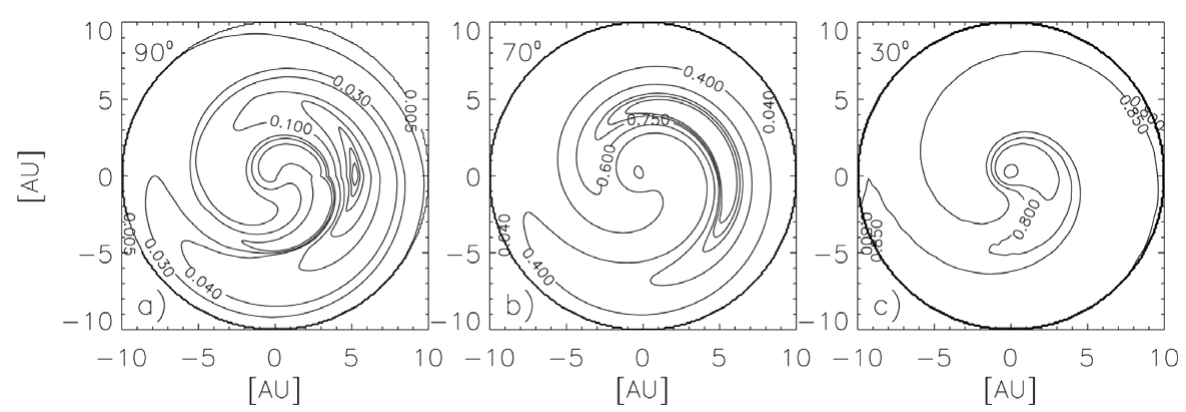

Figure 3. Three-dimensional distribution of Jovian electrons at different polar angles, as indicated.

Sun is assumed to rotate rigidly at the equatorial rate, differential rotation will cause a footpoint to move in heliomagnetic latitude and longitude, thus experiencing different degrees of non-radial expansion. The end result is a field line that moves in heliographic latitude.

Over the last ten years, various attempts to incorporate the Fisk field into numerical modulation models have been reported (Kóta \& Jokipii 1999; Burger et al. 2001; Burger $\&$ Hitge 2004). A recent overview of various models for the heliospheric magnetic field, including Fisk-type fields, is given by Burger (2005). Recently Burger et al. (2008) presented a Fisk-Parker hybrid field where at high latitudes the field is a mixture of Fisk field and Parker field, and in the equatorial region it is a pure Parker field. They confirmed the result of Burger \& Hitge (2004) that a Fisk-type heliospheric magnetic field provides a natural explanation for the observed linear relationship between the amplitude of the recurrent cosmic-ray variations and the global latitude gradient, first reported by Zhang (1997), and showed that this relationship holds for helium, protons, and electrons.

\section{Long-term cosmic ray modulation}

It was originally shown by Perko \& Fisk (1983) and le Roux \& Potgieter (1989) that cosmic ray modulation over long periods requires some form of propagating diffusion barriers. This is especially true for solar maximum when step decreases are observed. The largest form of these diffusion barriers are called global merged interaction regions (GMIRs) (Burlaga et al. 1993). Equally important are gradient, curvature and current sheet drifts (Jokipii et al. 1977) as confirmed by comprehensive modeling done by le Roux \& Potgieter (1995). These authors showed that it was possible to simulate, to the first-order, a complete 22-year modulation cycle by including a combination of drifts and GMIRs in a time-dependent modulation model. For typical solar minimum conditions, drifts together with changes in the current sheet are responsible modulation while toward solar maximum GMIRs caused the intensities to decrease in a step-like manner and drifts were less important due to the large tilt angles of the heliospheric current sheet.

More recently, Cane et al. (1999) and Wibberenz et al. (2002) argued that the step decreases observed at Earth could not be primarily caused by GMIRs because they occurred before any GMIRs could form beyond 10 AU. Instead they suggested that timedependent global changes in the heliospheric magnetic field might be responsible for longterm modulation. This was tested by Ferreira \& Potgieter (2004) who showed that indeed at neutron monitor energies, solar cycle related changes in the field magnitude alone can explain observations, but for lower energies this is no longer the case and a combination of these approaches were suggested. This can be done by scaling all the transport parameters 

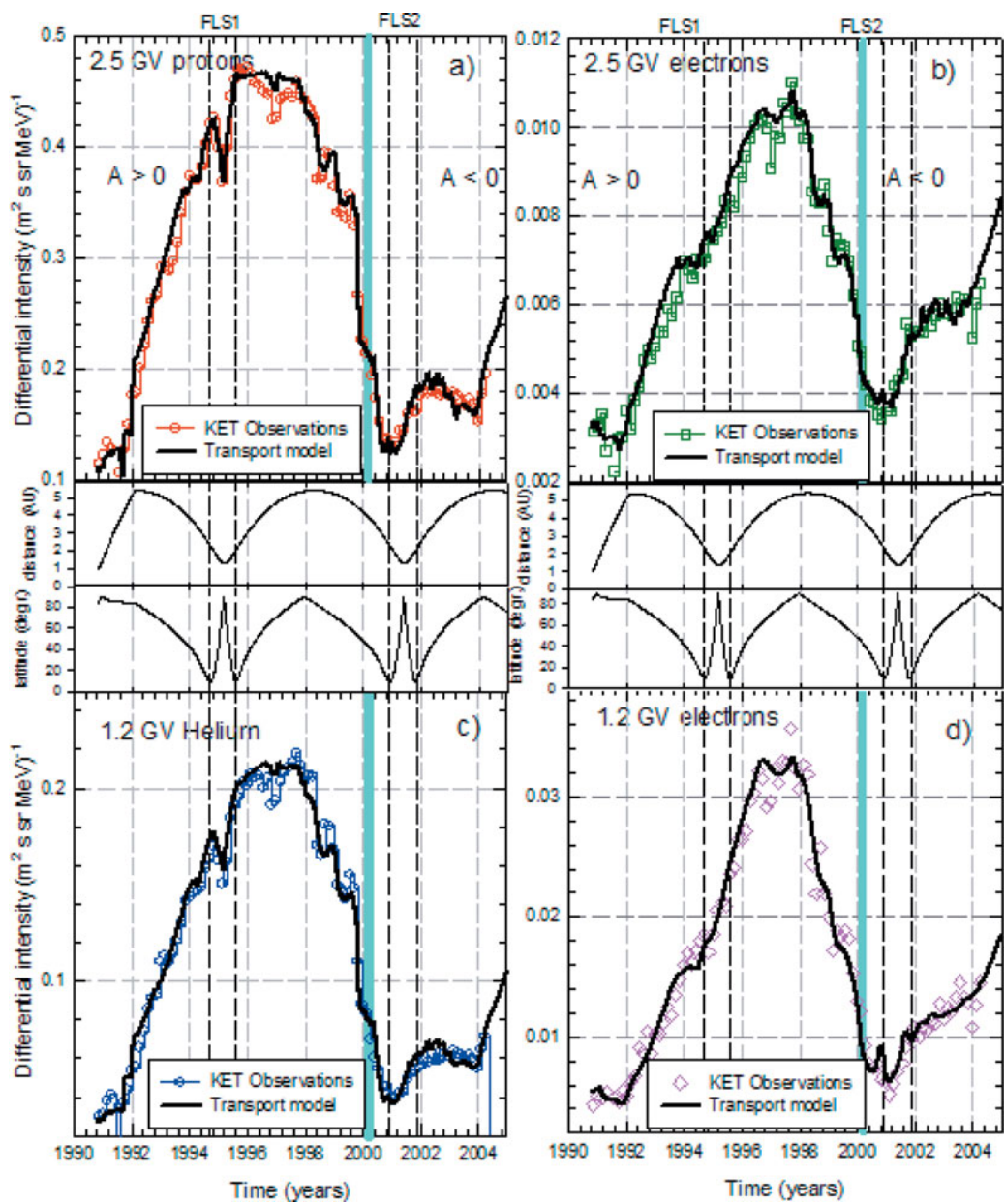

Figure 4. Model results compared to Ulysses/KET observations for protons, electrons and helium at different rigidities as indicated. The position in radial distance and latitude of the Ulysses spacecraft are also shown in the center panels. (from Ndiitwani et al. 2005).

with a function depending on the observed magnetic field at Earth and the current sheet tilt angle (Hoeksema 1992). This function results in diffusion coefficients which are roughly a factor of $\sim 10$ smaller for solar minima compared to solar maxima.

As shown by Ferreira \& Potgieter (2004), Ndiitwani et al. (2005) and Ferreira \& Scherer (2006) this approach incorporated in a numerical modulation model results in very realistic model computations when compared to spacecraft observations at various energies. An example is shown in Figure 4 (from Ndiitwani et al. 2005) showing model results compared to Ulysses/KET observations for protons, electrons and helium at different rigidities. Shown here is that the model could simulate the modulation amplitude between solar minimum and maximum correctly as well as step-decreases as they occur.

\section{References}

Baranov, V. B. \& Malama, Y. G. 1995, J. Geophys. Res., 100, 14755

Bell, A. R. 1978, MNRAS, 182, 147

Bieber, J. W., Matthaeus, W. H., Shalchi, A., \& Qin, G. 2004, Geophys. Res. Lett., 31, 10805 
Bieber, J. W., Matthaeus, W. H., Smith, C. W., Wanner, W., Kallenrode, M.-B., \& Wibberenz, G. 1994, ApJ, 420, 294

Blandford, R. D. \& Ostriker, J. P. 1978, ApJ, 221, L29

Borrmann, T. \& Fichtner, H. 2005, Adv. Sp. Res., 35, 2091

Burger, R. A. 2005, Adv. Sp. Res., 35, 636

Burger, R. A. \& Hitge, M. 2004, ApJ, 617, L73

Burger, R. A., Krüger, T. P. J., Hitge, M., \& Engelbrecht, N. E. 2008, ApJ, 674, 511

Burger, R. A., Potgieter, M. S., \& Heber, B. 2000, J. Geophys. Res., 105, 27447

Burger, R. A., van Niekerk, Y., \& Potgieter, M. S. 2001, Space Sci. Revs, 97, 331

Burlaga, L. F., McDonald, F. B., \& Ness, N. F. 1993, J. Geophys. Res., 98, 1

Cane, H. V., Wibberenz, G., Richardson, I. G., \& von Rosenvinge, T. T. 1999, Gephys. Res. Lett., 26, 565

Chenette, D. L., Conlon, T. F., \& Simpson, J. A. 1974, J. Geophys. Res., 79, 3551

Decker, R. B., Krimigis, S. M., Roelof, E. C., Hill, M. E., Armstrong, T. P., Gloeckler, G., Hamilton, D. C., \& Lanzerotti, L. J. 2005, Science, 309, 2020

Fahr, H. J., Kausch, T., \& Scherer, H. 2000, A\&A, 357, 268

Ferreira, S. E. S. \& Potgieter, M. S. 2004, ApJ, 603, 744

Ferreira, S. E. S., Potgieter, M. S., Burger, R. A., Heber, B., Fichtner, H., \& Lopate, C. 2001, J. Geophys. Res., 106, 29313

Ferreira, S. E. S., Potgieter, M. S., \& Scherer, K. 2007, J. Geophys. Res., 112, 11101

Ferreira, S. E. S. \& Scherer, K. 2006, ApJ, 642, 1256

Fichtner, H. 2005, Adv. Sp. Res., 35, 512

Fisk, L. A. 1971, J. Geophys. Res., 76, 221

Fisk, L. A. 1996, J. Geophys. Res., 101, 15547

Fisk, L. A. \& Gloeckler, G. 2006, ApJ, 640, L79

Fisk, L. A., Kozlovsky, B., \& Ramaty, R. 1974, ApJ, 190, L35

Fisk, L. A. \& Schwadron, N. A. 2001, ApJ, 560, 425

Florinski, V. \& Zank, G. P. 2006, Geophys. Res. Lett., 33, 15110

Florinski, V., Zank, G. P., \& Pogorelov, N. V. 2003, J. Geophys. Res., 108, 1

Forman, M. A. \& Jokipii, J. R. 1978, ApSS, 53, 507

Forman, M. A., Jokipii, J. R., \& Owens, A. J. 1974, ApJ, 192, 535

Friis-Christensen, E. \& Svensmark, H. 1997, Adv. Sp. Res., 20, 913

Giacalone, J. \& Jokipii, J. R. 1999, ApJ, 520, 204

Gleeson, L. J. \& Axford, W. I. 1967, ApJ, 149, L115

Hattingh, M. \& Burger, R. A. 1995, Adv. Sp. Res., 16, 213

Hoeksema, J. T. 1992, in Solar Wind Seven Colloquium, 191

Holzer, T. E. 1989, ARAA, 27, 199

Izmodenov, V., Malama, Y., \& Ruderman, M. S. 2005, A\&A, 429, 1069

Jokipii, J. R. 1966, ApJ, 146, 480

Jokipii, J. R., Levy, E. H., \& Hubbard, W. B. 1977, ApJ, 213, 861

Kallenbach, R., Hilchenbach, M., Chalov, S. V., Le Roux, J. A., \& Bamert, K. 2005, A\&A, 439,1

Kota, J. \& Jokipii, J. R. 1983, ApJ, 265, 573

Kóta, J. \& Jokipii, J. R. 1999, in Proc. 26th Int. Cosmic Ray Conf., Vol. 7, 9

Langner, U. W. \& Potgieter, M. S. 2005, ApJ, 630, 1114

Langner, U. W., Potgieter, M. S., Fichtner, H., \& Borrmann, T. 2006, J. Geophys. Res., 111, 1106

le Roux, J. A. \& Fichtner, H. 1999, J. Geophys. Res., 104, 4709

le Roux, J. A. \& Potgieter, M. S. 1989, Adv. Sp. Res., 9, 225

le Roux, J. A. \& Potgieter, M. S. 1990, ApJ, 361, 275

le Roux, J. A. \& Potgieter, M. S. 1995, ApJ, 442, 847

le Roux, J. A., Zank, G. P., Li, G., \& Webb, G. M. 2005, ApJ, 626, 1116

le Roux, J. A., Zank, G. P., \& Ptuskin, V. S. 1999, J. Geophys. Res., 104, 24845 
Lee, M. A. \& Fichtner, H. 2001, in The Outer Heliosphere: The Next Frontiers, ed. K. Scherer, H. Fichtner, H. J. Fahr, \& E. Marsch, 183

Lerche, I. \& Schlickeiser, R. 2001, A\&A, 378, 279

Matthaeus, W. H., Qin, G., Bieber, J. W., \& Zank, G. P. 2003, ApJ, 590, L53

McComas, D. J., Elliott, H. A., Schwadron, N. A., Gosling, J. T., Skoug, R. M., \& Goldstein, B. E. 2003, Geophys. Res. Lett., 30, 24

McComas, D. J., Goldstein, R., Gosling, J. T., \& Skoug, R. M. 2001, Space Sci. Revs, 97, 99

McComas, D. J. \& Schwadron, N. A. 2006, Geophys. Res. Lett., 33, 4102

Minnie, J., Bieber, J. W., Matthaeus, W. H., \& Burger, R. A. 2007, ApJ, 663, 1049

Minnie, J., Burger, R. A., Parhi, S., Matthaeus, W. H., \& Bieber, J. W. 2005, Adv. Sp. Res., 35,543

Moraal, H., Belov, A., \& Clem, J. M. 2000, Space Sci. Revs, 93, 285

Moraal, H. \& Potgieter, M. S. 1982, ApSS, 84, 519

Müller, H.-R., Frisch, P. C., Florinski, V., \& Zank, G. P. 2006, ApJ, 647, 1491

Ndiitwani, D. C., Ferreira, S. E. S., Potgieter, M. S., \& Heber, B. 2005, Annales Geophysicae, 23,1061

Opher, M., Stone, E. C., \& Liewer, P. C. 2006, ApJ, 640, L71

Paizis, C., et al. 1999, J. Geophys. Res., 104, 28241

Parker, E. N. 1958, ApJ, 128, 664

Parker, E. N. 1965, Planet. Space Sci., 13, 9

Parker, E. N. 2005, Space Weather, 3, S08004

Pauls, H. L. \& Zank, G. P. 1996, J. Geophys. Res., 101, 17081

Pauls, H. L. \& Zank, G. P. 1997, J. Geophys. Res., 102, 19779

Perko, J. S. \& Fisk, L. A. 1983, J. Geophys. Res., 88, 9033

Pogorelov, N. V., Zank, G. P., \& Ogino, T. 2006, ApJ, 644, 1299

Potgieter, M. S. 1998, Space Sci. Revs, 83, 147

Qin, G., Matthaeus, W. H., \& Bieber, J. W. 2002, Geophys. Res. Lett., 29, 7

Scherer, K. \& Fahr, H. J. 2003a, Ann. Geophys., 21, 1303

Scherer, K. \& Fahr, H. J. 2003b, Geophys. Res. Lett., 30, 17

Scherer, K. \& Ferreira, S. E. S. 2005, ASTRA, 1, 17

Scherer, K., et al. 2006, Space Sci. Revs, 127, 327

Scherer, K., Fichtner, H., Ferreira, S. E. S., Büsching, I., \& Potgieter, M. S. 2008, ApJ, 680, L105

Shalchi, A. 2006, A\&BA, 453, L43

Shalchi, A., Bieber, J. W., \& Matthaeus, W. H. 2004, ApJ, 604, 675

Shalchi, A., Bieber, J. W., \& Matthaeus, W. H. 2008, A\&BA, 483, 371

Shaviv, N. J. 2003, New Astronomy, 8, 39

Shaviv, N. J. \& Veizer, J. 2003, GSA Today, 13, 4

Simpson, J. A., et al. 1995, Science, 268, 1019

Simpson, J. A., Hamilton, D. C., McKibben, R. B., Mogro-Campero, A., Pyle, K. R., \& Tuzzolino, A. J. 1974, J. Geophys. Res., 79, 3522

Simpson, J. A., Zhang, M., \& Bame, S. 1996, ApJ, 465, L69

Stawicki, O. 2005a, Adv. Sp. Res., 35, 547

Stawicki, O. 2005b, ApJ, 624, 178

Steenberg, C. D. \& Moraal, H. 1996, ApJ, 463, 776

Stone, E. C., Cummings, A. C., McDonald, F. B., Heikkila, B. C., Lal, N., \& Webber, W. R. 2005, Science, 309, 2017

Suess, S. T. 1990, Rev. Geophys., 28, 97

Svensmark, H. 1998, Phys. Rev. Lett., 81, 5027

Teufel, A. \& Schlickeiser, R. 2002, A\&A, 393, 703

Washimi, H. \& Tanaka, T. 1996, Space Sci. Revs, 78, 85

Webb, G. M. \& Gleeson, L. J. 1979, ApSS, 60, 335

Webb, G. M., Zank, G. P., Kaghashvili, E. K., \& le Roux, J. A. 2006, ApJ, 651, 211

Wibberenz, G., Richardson, I. G., \& Cane, H. V. 2002, J. Geophys. Res., 107, 5 
Zank, G. P. \& Müller, H.-R. 2003, J. Geophys. Res., 108, 7

Zhang, M. 1997, ApJ, 488, 841

Zhang, M. 2006, in AIP Conf. Proc. 858: Physics of the inner heliosheath: 5th Annual IGPP International Astrophysics Conference, ed. J. Heerikhuisen \& et al., 226

\section{Discussion}

FISK: I think there is a fundamental problem with your stochastic acceleration mechanism to create the CRs in the heliosheath. You are using a damping mechanism, yet there is inadequate energy in the turbulence in the heliosheath to provide the energy in the ACRs.

FERREIRA: You are certainly correct. We studied stochastic acceleration of ACRs when Voyager1 just crossed the boundary. Recent observations do yield more energy in PUIs than to the magnetic field than expected. Therefore, indeed our model does overestimate the effect and we are busy looking deeper into this.

ANONYMOUS: 1) How strong is the GMIR argument for producing the step decreases, given the fact that a GMIR didn't form during the Halloween 2003 storms. 2) Why don't you consider the difference between high latitude and low latitude CMEs in the $\mathrm{A}>0$ cycle?

FERREIRA: There is evidence that GMIRs do cause the 11-year cycle, at least toward solar maximum. However this is only one aspect, with HMF and current sheet tilt angle also causing time-dependent modulation.

Cliver: Can you tell us about the current level for cosmic ray intensity? Because the solar polar fields are quite weak at this minimum, is the GCR intensity significantly higher than in previous minima?

FERREIRA: The recent Ulysses/KET data will be published soon. I can show you now one of Bernd Heber's slides.

MelnikOv: How important is the pitch angle scattering which was not included in the transport equation?

FERREIRA: It is important for second-order effects. We are more interested in global modulation. For work on this concerning cosmic rays see, e.g., Florinski and Le Roux and co-workers. 